\title{
Inhibitory Effects of Human-Derived Bifidobacterium on Pathogenic Escherichia coli Serotype O-111
}

\author{
Tomoko Araya-KojIma, Tomoko Yaeshima, Norio Ishibashi, \\ Seiichi Shimamura and Hirotoshi Hayasawa \\ Nutritional Science Laboratory, Morinaga Milk Industry, Co., Ltd., 1-83, Higashihara \\ 5-chome, Zama, Kanagawa, 228 Japan
}

(Received for publication, February 29, 1996)

\begin{abstract}
The inhibitory effects of seven human-derived Bifidobacterium strains on pathogenic Escherichia coli serotype O-111 were examined in co-cultivation. All of the Bifidobacterium strains tested inhibited the growth of $E$. coli and limited the increase in the ammonia concentration in the co-cultures. Also, in each instance, the $\mathrm{pH}$ of the culture medium decreased due to their production of acetic and lactic acids. From the results of $\mathrm{pH}$-controlled fermentation, the growth inhibition of $E$. coli was attributed mainly to the decrease in the $\mathrm{pH}$ of the culture medium. The possibility that some unidentified inhibitory substances derived from Bifidobacterium strains were present was little thought. In mono-cultures of Bifidobacterium strains, the ammonia concentration in the culture medium decreased to a level below that initially present in the medium prior to inoculation. Enzymatic assays showed that the activity of an enzyme involved in ammonia production (L-asparagine deaminase) was much weaker in each of the Bifidobacterium strains than in E. coli, whereas the activities of enzymes involved in ammonia assimilation (glutamine synthetase, glutamate synthase and glutamate dehydrogenase) were much higher in bifidobacteria. The decrease in the ammonia concentration in the co-culture appears to be related not only to the number of colonyforming units of bifidobacteria but also to the differences among the bacteria examined in enzymatic activities which are concerned with utilization and production of ammonia.
\end{abstract}

Key words: bifidobacteria; Escherichia coli; O-111; inhibition ammonia

Bifidobacteria comprise a major group in the human and animal intestinal flora along with bacteroides and eubacteria. This genus is divided into 28 species, including seven species isolated from the human intestine: $B$. breve and $B$. infantis, isolated mainly from babies and $B$. adolescentis, $B$. longum, B. bifidum, B. catenulatum and B. pseudocatenulatum, isolated mainly from adults $(6)$. In the large intestine, bifidobacteria produce acetic and lactic acids and is thought to inhibit the proliferation of putrefactive bacteria such as escherichiae, clostridia and eubacteria, and inhibit the synthesis of harmful products (reviewed in 5). Recently, reports were presented which attributed these inhibitory effects of bifidobacteria to both the decrease in the $\mathrm{pH}$ of the culture medium and the produced acetic and/or lactic acids themselves (4), or to some unidentified inhibitory substance derived from bifidobacteria (3).

Here, the inhibitory effects of seven humanderived Bifidobacterium strains on pathogenic $E$. coli serotype O-111 were examined in co-cultivation by measuring the number of bacterial colony-forming units (GFUs), the $\mathrm{pH}$ and the ammonia concentration of the culture medium. Ammonia is known to be one of the harmful substances pro- 
duced by intestinal bacteria. Assays of several bacterial enzymes concerned with changes in the ammonia concentration were also performed.

\section{MATERIALS AND METHODS}

Bacterial strains. B. adolescentis ATCG $15703^{\mathrm{T}}$, B. catenulatum ATCC 27675, B. bifidum ATCC 15696, B. breve ATCC $15700^{\mathrm{T}}$, B. infantis ATCC $15697^{\mathrm{T}}$ and $B$. longum ATCC $15707^{\mathrm{T}}$ were purchased from the American Type Culture Collection. B. pseudocatenulatum M101-4 was a gift from Prof. T. Mitsuoka (8). Escherichia coli serotype O111 was obtained from the Institute of Medical Science, The University of Tokyo. Hereafter, each strain is represented by its species name.

Culture conditions. Cultivation was performed as previously described ( 1 ) except in $\mathrm{pH}$-controlled fermentation. $\mathrm{pH}$-controlled cultivation was performed using a jar fermenter (Mini Jar Fermenter Model MB-C, Iwashiya A.D.M. Co., Ltd., Shinagawa, Japan) with continuous stirring at $90 \mathrm{rpm}$ and supplying with $\mathrm{CO}_{2}$ gas continuously. CFUs were counted as previously described (1).

Preparation of cell suspensions and cell-free extracts. Cell suspensions and cell-free extracts were prepared as previously described (I).

Assays. Concentrations of acetic and lactic acids in the culture were assayed using F-Kits (Boehringer Mannheim).

Ammonia and protein concentrations were assayed as previously described (1).

Urease and deaminases for $20 \mathrm{~L}$-amino acids were assayed at $\mathrm{pH} 7.0$ as previously described (1). One unit of urease or deaminase activity was defined as the amount that produces one micromole of ammonia per minute. Specific activities are shown as the activity per one $\mathrm{OD}_{660}$ unit per milliliter of cell suspension.

Glutamate dehydrogenase (GDH), glutamate synthase (GOGAT), and Glutamine synthetase (GS) were assayed as previously described (1). The reaction $\mathrm{pH}$ was 7.5 for GDH and GOGAT assays, and 6.5 for GS assay. One unit of GDH or GOGAT activity was defined as the amount that oxidizes one micromole of NADPH per minute. One unit of GS activity was defined as the amount that synthesizes one micromole of $\gamma$-glutamylhydroxamate per minute. Specific activities of these three enzymes are shown as the activity per milligram of protein in the cell-free extract.

Measurement of $\mathrm{pH} . \mathrm{pH}$ in the culture media was measured using a model F-13 $\mathrm{pH}$ meter (Horiba, Japan).

Note. All experiments were performed in duplicate and means of duplicates are presented in figures and tables.

\section{RESULTS}

Co-Cultivation with Seven Human-Derived Bifidobacterium Strains and E. coli Serotype O-111

To examine the inhibitory effects of Bifidobacterium on the growth of $E$. coli, co-cultivation was performed for $24 \mathrm{hr}$ and samples were taken at 4-hr intervals for measurement of $\mathrm{pH}$, bacterial CFUs and ammonia concentration. The inoculation ratio of each Bifidobacterium strain to $E$. coli O-111 was mixed up to about $1: 1$. Mono-cultivation of these strains was also performed as the control. The results are shown in Fig. 1 and Table 1. In all cases, the growth curves of $E$. coli in cocultivation with bifidobacteria (Fig. 1 from A to G) showed different patterns when compared with that obtained in mono-cultivation (Fig. $1 \mathrm{H}$ ), whereas the growth curves of the Bifidobacterium strains were similar to those in the mono-cultures (Fig. 1 from A to G). With each Bifidobacterium strain tested, the CFU of $E$. coli after incubation for $24 \mathrm{hr}$ decreased to a level below $30 \%$ of that observed in mono-culture (Table 1). The ammonia concentration was lower than that in monoculture in each case with the exception of $B$. catenulatum (Fig. 1B, Table 1). These inhibitory effects, especially the decrease in CFU of E. coli, seemed to depend on the lowering of the $\mathrm{pH}$ of the culture medium. E. coli cannot grow below 4.5 (2), and the survival ratio was higher when the $\mathrm{pH}$ of the co-culture was above 4.5 , as with $B$. catenulatum and $B$. infantis (Table 1). The decrease in the ammonia concentration seemed to depend not only on the lowering of $\mathrm{pH}$ and/or the resulting decrease in CFU of $E$. coli but also on the increase in CFU of Bifidobacterium because the ex- 

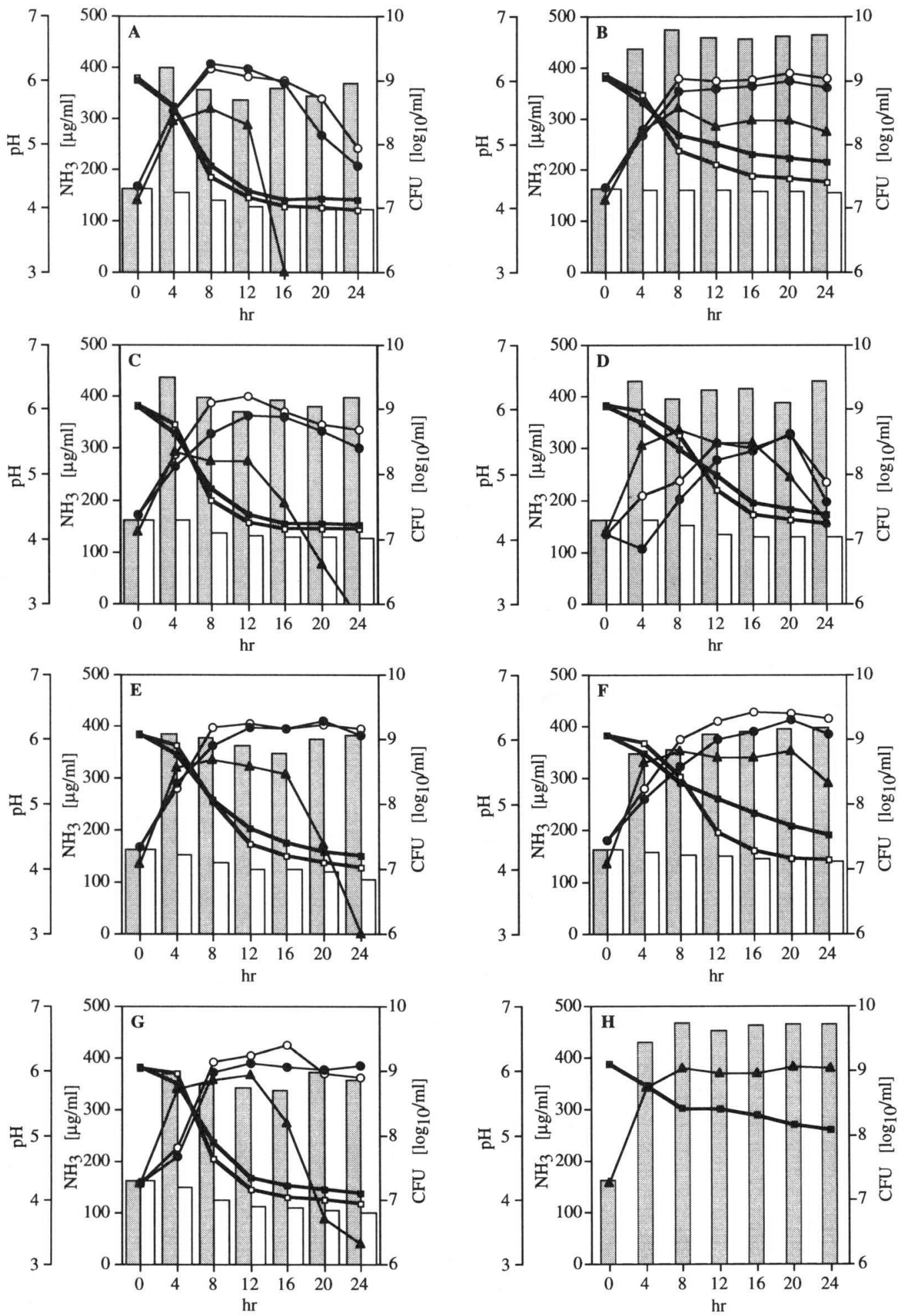

Fig. 1. Growth of E. coli and (A) B. adolescentis, (B) B. catenulatum, (C) B. pseudocatenulatum, (D) B. bifidum, (E) B. breve, $(\mathrm{F})$ B. infantis and $(\mathrm{G})$ B. longum in co-cultures, and $(\mathrm{H})$ E. coli in mono-culture as a control.

From (A) to (G), data of each Bifidobacterium strain in mono-culture are also shown. CFUs of each Biffdobacterium strain in co-culture (--) and in mono-culture (-O-), CFU of E. coli $(-\mathbf{-}-), \mathrm{pH}$ of co-culture and $E$. coli

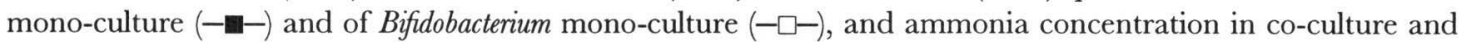
E. coli mono-culture $(\square)$ and in Bifidobacterium mono-culture $(\square)$ were measured at the times indicated. 
Table 1. Decrease in CFU of $E$. coli and ammonia concentration in co-culture medium with $E$. coli and Bifidobacterium after $24 \mathrm{hr}$ of incubation ${ }^{\mathrm{a}}$

\begin{tabular}{lccc}
\hline $\begin{array}{c}\text { Strain cultured } \\
\text { with } \text { E. coli }\end{array}$ & $\mathrm{pH}$ & $\begin{array}{c}\text { CFU' }^{\mathrm{b}} \\
(\%)\end{array}$ & $\begin{array}{c}\text { Ammonia }^{\mathrm{c}} \\
(\%)\end{array}$ \\
\hline B. adolescentis & 4.12 & 0.00 & 71.01 \\
B. catenulatum & 4.71 & 31.45 & 100.00 \\
B. pseudocatenulatum & 4.22 & 0.00 & 81.27 \\
B. bifidum & 4.37 & 3.83 & 91.77 \\
B. breve & 4.19 & 0.11 & 72.84 \\
B. infantis & 4.52 & 22.17 & 77.67 \\
B. longum & 4.09 & 0.19 & 64.67 \\
No strain & 5.15 & $100^{\mathrm{b}}$ & $100^{\mathrm{c}}$ \\
\hline
\end{tabular}

a The data used here were the values shown in Fig. 1.

b \% of the CFU of $E$. coli after $24 \mathrm{hr}$ of cultivation in the co-culture when the CFU of $E$. coli in the monoculture was taken as $100 \%$.

c\% of the increase in the ammonia concentration after $24 \mathrm{hr}$ of cultivation in the co-culture when the increase in the ammonia concentration in the $E$. coli mono-culture was taken as $100 \%$.

tent of inhibition of ammonia production was higher with $B$. infantis, in which case the $\mathrm{pH}$ was above 4.5 and the CFU of $B$. infantis was above $10^{9} \mathrm{CFU} / \mathrm{ml}$ (Fig. 1F), than it was with B. pseudocatenulatum or $B$. bifidum, in which cases the $\mathrm{pH}$ was below 4.5 but the CFU of each Bifidobacterium strain was below $10^{9} \mathrm{CFU} / \mathrm{ml}$ (Fig. 1C, D).

pH-Controlled Co-Cultivation of B. longum and E. coli

As mentioned above, the growth inhibition of $E$. coli seemed to be due to the decrease in the $\mathrm{pH}$ of the culture medium. However, the potential influence of the produced acetic and/or lactic acids themselves or of some unidentified inhibitory substances derived from bifidobacteria could not be excluded. In order to confirm that the decrease in CFU of $E$. coli in co-cultures results from the lowering of $\mathrm{pH}$, co-cultivation of $B$. longum and $E$. coli was performed under $\mathrm{pH}$-controlled conditions. $B$. longum was chosen for its strong ability to lower the $\mathrm{pH}$ of the culture medium. The results are shown in Fig. 2. Comparing the results obtained under $\mathrm{pH}$-controlled (Fig. 2A, C) and $\mathrm{pH}$-uncontrolled (Fig. 2B, D) conditions, the ammonia concentration in the culture medium was similar (Fig. 2A, B) and the molar ratios of the
Table 2. Activities of ammonia-assimilating enzymes

\begin{tabular}{lccc}
\hline \multicolumn{1}{c}{ Strain } & $\begin{array}{c}\text { GS } \\
{[\mathrm{U} / \mathrm{mg}]}\end{array}$ & $\begin{array}{c}\text { GOGAT } \\
{[\mathrm{mU} / \mathrm{mg}]}\end{array}$ & $\begin{array}{c}\text { GDH } \\
{[\mathrm{U} / \mathrm{mg}]}\end{array}$ \\
\hline B. adolescentis & 4.71 & 38.64 & 1.42 \\
B. catenulatum & 0.63 & 64.64 & 0.47 \\
B. pseudocatenulatum & 3.20 & 30.46 & 0.72 \\
B. bifidum & 1.84 & 51.27 & 1.16 \\
B. breve & 0.66 & 77.27 & 5.22 \\
B. infantis & 1.33 & 66.13 & 1.04 \\
B. longum & 8.24 & 40.12 & 2.93 \\
E. coli & 0.03 & 2.09 & 0.30 \\
\hline
\end{tabular}

acetate and lactate concentrations after $24 \mathrm{hr}$ of cultivation were 1.09 and 0.74 , respectively, by defining the values under $\mathrm{pH}$-uncontrolled condition as 1.0 (Fig. 2C, D), whereas the growth curve patterns of both $E$. coli and $B$. longum were completely different (Fig. 2A, B). The CFU of $E$. coli was always higher than that of $B$. longum under $\mathrm{pH}$-controlled conditions and, conversely, the CFU of $B$. longum was always higher than that of $E$. coli under $\mathrm{pH}$-uncontrolled conditions. The GFU of B. longum reached a maximum of $5 \times 10^{8} \mathrm{CFU} / \mathrm{ml}$ in both instances.

\section{Activities of Enzymes Which Affect the Ammonia Concen- tration}

It was shown that in mono-cultures of Bifidobacterium the ammonia concentration of the culture medium decreased to a level below that present in the medium prior to inoculation (Fig. 1, from A to G). Since in the mono-cultivations of $B$. catenulatum (Fig. 1B) and B. infantis (Fig. 1F) the decrease in ammonia was smaller than that with B. breve (Fig. 1E) and B. longum (Fig. 1G) although the CFU values were similar, the activities of enzymes which assimilate or produce ammonia were considered to be important. Therefore, the activities of enzymes known to assimilate (Table 2) and produce (Table 3 ) ammonia were examined. In Table 2, the activities of GS and GDH differed according to the magnitude of the decrease of the ammonia concentration in the culture medium (Fig. 1, from $A$ to $G$ ). The contribution of GOGAT activity was rather unclear. In Table 3 , the activities of ammonia-producing enzymes were rather 

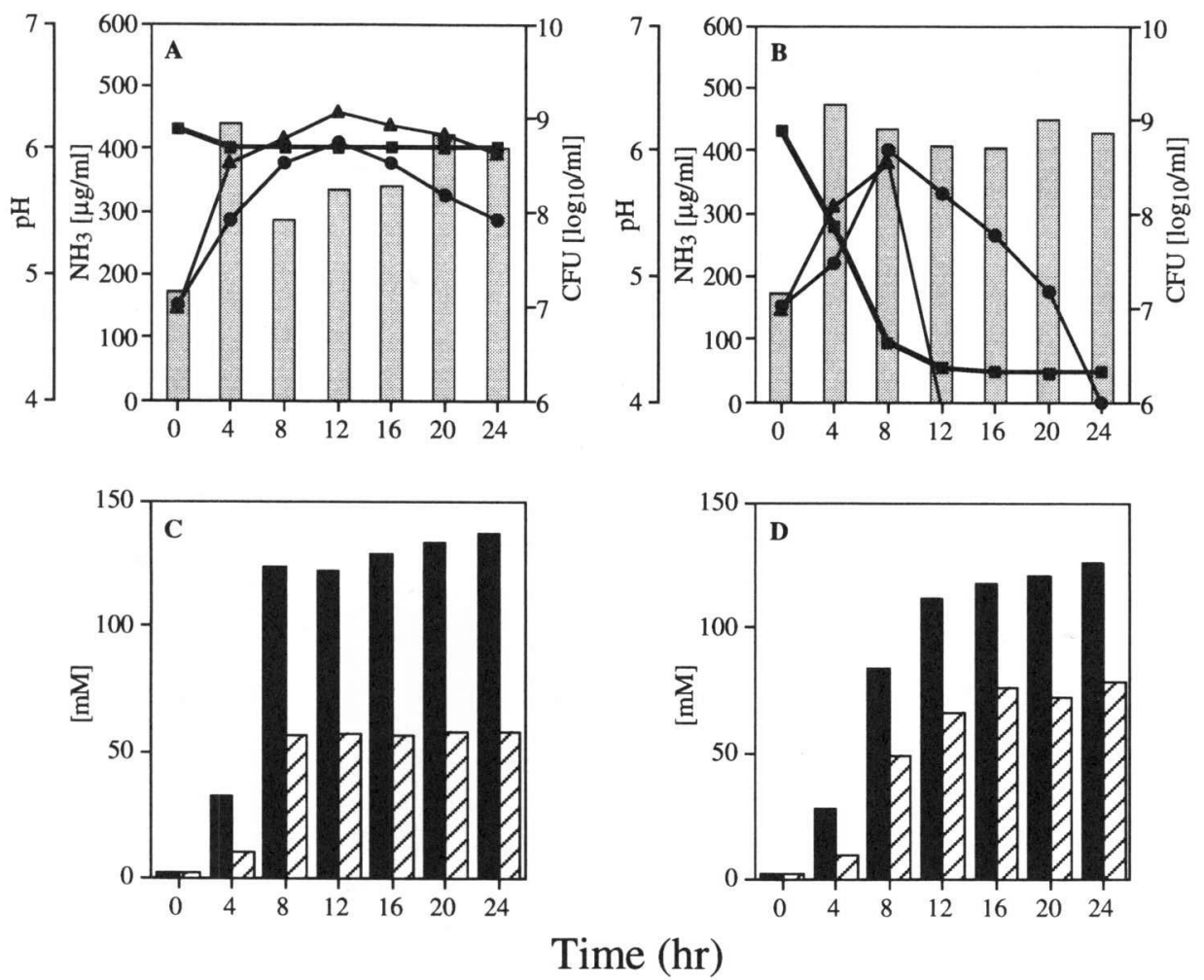

Fig. 2. (A, B) Growth of E. coli and B. longum in co-cultures under (A) pH-controlled and (B) pH-uncontrolled conditions. Marks used are the same as in Fig. 1.

$(\mathrm{C}, \mathrm{D})$ End product formation in co-cultures under $(\mathrm{C}) \mathrm{pH}$-controlled and (D) $\mathrm{pH}$-uncontrolled conditions. Acetic acid ( and lactic acid (חD) concentrations were measured at the times indicated.

Table 3. Distribution of ammonia-producing enzymes ${ }^{\mathrm{a}}$

\begin{tabular}{lcccccc}
\hline \multirow{2}{*}{ Strain } & \multicolumn{7}{c}{ Deaminase [mU/OD unit ml] } \\
\cline { 2 - 6 } & Asn & Asp & Gln & Ile & Leu & Lys \\
\hline B. adolescentis & 3.53 & - & 16.64 & - & - & 1.32 \\
B. catenulatum & 1.25 & - & 17.15 & - & - & 1.22 \\
B. pseudocatenulatum & 4.03 & - & 20.16 & - & - & 1.37 \\
B. bifidum & - & - & 15.38 & - & - & - \\
B. breve & - & - & 13.19 & - & - & - \\
B. infantis & - & - & 13.50 & 1.19 & 1.44 & 1.48 \\
B. longum & - & - & 16.63 & 1.00 & - & - \\
E. coli & 99.05 & 2.23 & 15.48 & - & - & 2.80 \\
\hline
\end{tabular}

a Though urease and deaminases for 20 L-amino acids were assayed as ammonia-producing enzymes, enzymes whose activities were not detected in any strain are omitted.

similar among Bifidobacterium species, but a greater number of enzymes were active in $B$. catenulatum and $B$. infantis than in B. breve and B. longum. E. coli was found to have much lower activities of the ammonia-assimilating enzymes (Table 2) and higher activity of an enzyme involved in ammonia production (L-asparagine deaminase) (Table 3) compared with the Bifidobacterium strains tested.

\section{DISCUSSION}

In this study, the inhibitory effects of seven human-derived Bifidobacterium species on a pathogenic strain of $E$. coli was examined. Our results demonstrate that at least in the case of $B$. longum 
the growth inhibition of $E$. coli was attributed mainly to the lowering of $\mathrm{pH}$ and, to some extent, to the produced acetic and lactic acids themselves. The findings that support this conclusion are the following. First, the molar ratio of the lactate concentration under $\mathrm{pH}$-controlled and -uncontrolled conditions after $24 \mathrm{hr}$ of cultivation was 0.74 , taking the value under the $\mathrm{pH}$-uncontrolled condition as 1.0 , and it might be involved in the difference in inhibition. Second, and more fundamentally, as the $\mathrm{pH}$ of the medium decreases, the $\Delta \mathrm{pH}$ across the cytoplasmic membrane becomes larger, and as a result the cells might tend to accumulate acetic and lactic acids to a greater extent. So the effects of these acids in bacterial cells cannot be excluded. Third, the production of some unidentified inhibitory substance by Bifidobacterium seems unlikely, unless such a substance is produced only after the $\mathrm{pH}$ of the culture medium decreased, since the maximum CFU of $B$. longum was similar under $\mathrm{pH}$-controlled and -uncontrolled conditions.

Ammonia induces hepatic encephalitis and coma in patients with cirrhosis or other liver diseases when it exists at a high concentration in the human body (7). Bifidobacteria have been thought to play a role in reducing the levels of harmful substances including ammonia in the gastrointestinal tract by inhibition of intraintestinal ammonia-producing bacteria (5). From our results, the decrease in the ammonia concentration in the culture medium is thought to depend neither on the lowering of $\mathrm{pH}$ nor on the decrease in CFU of E. coli (Fig. 2A, B). Rather, it appears to depend on the GFU of each Bifidobacterium strain
(Fig. 1) and the differences observed among the bacteria tested in the activities of enzymes which assimilate (Table 2) and produce (Table 3) ammonia. Though the participation of GOGAT was not suggested, this enzyme might be unimportant since it does not directly consume ammonia while GS and GDH do.

\section{REFERENCES}

(1) Araya-Kojima T, Yaeshima T, Ishibashi N, Shimamura $\mathrm{S}$ and Hayasawa $\mathrm{H}$ : Inhibitory effects of Bifidobacterium longum BB536 on harmful intestinal bacteria. Bifidobacteria Microflora 14: 59-66, 1995

(2) Davis BD: Bacterial nutrition and growth. In Microbiology - Including Immunology and Molecular Genetics, Davis BD, Dulbecco R, Eisen $H N$ and Ginsberg HS eds, 3rd ed, Harper \& Row Publishers, Inc., Philadelphia, 1980, p. 59-70

(3) Gibson GR and Wang X: Regulatory effects of bifidobacteria on the growth of other colonic bacteria. J Appl Bacteriol 77: 412-420, 1994

(4) Ibrahim SA and Bezkorovainy A: Inhibition of Escherichia coli by bifidobacteria. J Food Protect 56: 713-715, 1993

(5) Mitsuoka T: Intestinal Bacteria and Health-An Introductory Narrative, Harcourt Brace Jovanovich Japan, Inc., Tokyo, 1978

(6) Mitsuoka T: Classification of bifidobacteria. In Studies of Bifidobacteria, Mitsuoka T ed, Japan Bifidus Foundation, Tokyo, 1994, p. 23-29 (in Japanese)

(7) Podolsky DK and Isselbacher KJ: Cirrhosis. In Harrison's Principles of Internal Medicine, Braunwald E et al eds, 11 th ed, McGraw-Hill, New York, 1987, p. 1349

(8) Yaeshima T, Fujisawa T and Mitsuoka T: Bifidobacterium species expressing phenotypical similarity to Bifidobacterium adolescentis isolated from the feces of human adults. Bifidobacteria Microflora 11: 25-32, 1992 